\title{
EL GENOMA DEL FRIJOL
}

CAROLA ESCOBAR,

DAVID TALLEDO,

TERESA NAMISATO.

Laburabeis oe Biologis Celelar y Gienetick Seccios de Cinogentica.

Facultad de Ciencias Blelólons

Universidod Ricando Paima

Aptida 138,Lime-PERU

\section{RESUMEN}

\begin{abstract}
El artícule gresenta un andilisis comparative de bos cariotipos de cuairo especies del género Phaseolius L, una de ellas evidenciada pee primern vez en el preseate trabajo. 5 e devcriben los cromosonas de cada cariocipo en función đe sa longirud relstiva (L/). indice centromérico (iv) y oeras peculiaridades individuales y se propone un sistema para clasificarlos. En base a las observaciones realizadas y a la revisión de los. resultados de otros inventigadores se sugiere el nömero base $(x=11)$ de ctumwocmas para las especies del gtacero.
\end{abstract}

\section{SUMMARY}

The article presents a comparative analysis of the karyotypes of four species of Phasenlas La; one of them is reparied by the first time. This poblicasios describes the chromosomes of each karyotype according to its relative longitude ( $2 /$ ); eencromeric ratio ( $\mathrm{P}$ ) and other individual features, and a classification system is proposed. Accarding to the results and bibtiograghical revisice, the chromceomal base namber ( $x i 11$ ) in this species is suzpested.

KEY WORDS; Phexpolus, chromosomes, karyotypes.

\section{INTRODUCCION}

Entre las leguminosias de la flora mundial existen 7 géneros importantes: Cicer; Lens; Lupinus; Medicago; Phaseolus; Pisum y Vicia, siendo sin lugar a dudas el género Phaseolus $(2 n=22)$ el de mayor relevancia en América Latína, con 4 especies anuales de importancia económica: $P$ h, acuajfolius Gray, Ph. coccinews L., Ph, hunatus $\mathrm{L}$ y $P$ h. vulgaris $\mathrm{L}$.

Para las especies del género Phaseolus existen suficientes investigaciones en cuanto a su dispersión, valor alimenticio y cruzamientos interespecificos. Sin embargo, los trabajos cariológicos sobre estos cultivos son escasos y se limitan fundamentalmente a informar sobre el número cromosómico, careciendo casi totalmente de caracterizaciones cariotipicas, a pesar que estas especies son consideradas como cultivos con prioridad uno para trabajos en recursos genéticos en América Latina (Holle, 1984).

Recientes investigaciones en $P h$. vulgaris L (Turkov y col; 1988), Ph. acutifolius Gray, Ph. coccineus L. y Ph. Lunatus L. (Escobar y col:; 1989, 1991, 1992) reportan las fórmulas cariotípicas de estas especies y corroboran la presencia de 11 pares de cromosomas pequetios. Sin cmhargo, un análisis cariomorfológico que permita comparar los cariotipos de especies del género Phaseolus y estudiar su polimorfismo cromosómico puede facilitar tanto la utilización de los métodos del fítomejoramiento analítico como la elucidación de la senda evolutiva del género. La dificultad para evidenciar los cromosomas de estas especies se debe principalmente a su pequeño tamaño, comprendido entre 2 y 4 micras. En la presente investigación se utiliza una metodologia que incluye el pre-tratamiento con inhibidores mitóticos especificos y bajas temperaturas positivas, lo que hace posible una mejor diferenciación de los cromosomas. Se presenta un artálisis paramétrico completodecadacumosuma, decerminando su longitud absoluta, longitud relativa e f́ndice centromérico, asi como el índice de espiralización de cada complemento con el propósito de evidenciar los caracteres morfolḱgicos de los cromosomas de cada colección estudiada y facilitar la caracterización de las mismas utilizando criterios objetivos y cuantificables.

El desarrollo de métodos para el análisis cariomorfologico en las 4 especies más importantes de Phaseolus puede contribuir a la elucidación de la senda evolutiva del género y brindar un aporte de importancia príctica que sirva como base en la elaboración de programas de fitomejoramiento genético, considerando la relación existente entre la morfologfa cromosómica de los gametos de los progenitores $y$ la fertilidad de los hibridos.

\section{MATERIALES Y METODOS}

Para cada una de las especies estudiadas se usaron 50 semillas certificadas. Las semillas fueron proporcionadas por el Banco de Semillas del Instituto Nacional de 
Investigaciones para la Agricultura (INIA), filial de Ica, El trabajo se desarrolló en condiciones de laboratorio, fluctuando la temperatura de éste entre $18-20^{\circ} \mathrm{C}$ en los meses de invierno y $22.28^{\circ} \mathrm{C}$ en los meses de verano.

Las semillas fueron colocadas en placas Petri sobre papel filtro humedecido, a temperatura ambiente. Para las coloraciones se tomaron raices secundarias de 1.0 - 1.5 $\mathrm{cm}$. de longitud, tratándolas con una solución acuosa de $A B N$ al $1 \%$, a bajas temperaturas positivas durante 2:30 hrs. Luego se procedio a fijarlas en una solución fresca de Carnoy, posteriormente fueron coloreadas con orceina acética según métodos usuales (O'Mara, 1948; Tjio y Levan, 1954). Las microfotografias fueron tomadas en un microscopio con cámara incorporada (Nikon) utilizando una película de baja sensibilidad (Mikrat 300. ASA 1) a 800 aumentos.

Para el análisis cariomorfológico fueron scleccionadas 10 metafases por especie que presentaron indices de espiralización similares con un margen de error del $10 \%$, según lo recomendado por Sasaki en 1961, Las microfotografías fueron ampliadas hasta un aumento total de 4,000X. Los parámetros morfométricos (i.e.: Longitud absoluta - L*, Indice centromérico - $\mathrm{F}_{-}$, Longitud relativa $-L$ ) fueron calculados a partir de mediciones de los cromosomas realizadas sobre las ampliaciones de las microfotografias.

En base a estos resultados para cada especie se plantearon las fórmulas cariotípicas: $\mathbf{K}(\mathbf{n}=)=\mathbf{M}+\mathbf{S M}+\mathbf{A}$. donde $\mathrm{K}=$ cariotipo, $\mathrm{n}=$ complemento haploide y $\mathrm{M}, \mathrm{SM}$ y A significan metacéntricos, sub-metacéntricos y actocéntricos, respectivamente. Estas fómulas se plantean por primera vex.

\section{RESULTADOS}

\section{Phaseolus acurifolius}

Para esta especie se evidenció la fórmula cariotípica: $K(n=11)=7 M+4 S M$.

Los indices centroméricos (Is) para los pares 1-7 son similares, presentándose en un rango comprendido entre $43.19 \%$ para el par 1 y $49.56 \%$ para el par 6 . Si tomamos en cuenta las longitudes relativas ( $\mathrm{L}^{3}$ ) observaremos similitud para los pares $1-2\left(\mathrm{~L}_{1}=11.65 \%, \mathrm{~L}_{2}^{r}=11.34 \%\right)$, Los pares 3,4 y 5 presentan diferencias tanto entre sí como con respecto a los demás metacéntricos. Las $\mathrm{L}^{r}$ de los pares $6-7$ son iguales $\left(\mathrm{L}^{\prime},=7.26 \%, \mathrm{~L}_{7}^{\prime}=7.26 \%\right)$.

Entre los sub-metacéntricos encontramos 1 que varian entre $35.78 \%$ y $33.23 \%$, para los pares 9 y 11 respectivamente; mientras que las $\mathrm{L}^{\prime}$ varian significativamente para los pares 8 y $9\left(\mathrm{~L}_{s}=11.30 \%, \mathrm{~L}_{\mathrm{s}}=8,65 \%\right)$ y son similares para los pares 10 y $11\left(\mathrm{~L}_{10}^{*}=7.84 \%, \mathrm{~L}_{10}^{*}=7.72 \%\right)$.

En base a los parámetros morfométricos registrados es posible identificar los pares $3,4,5,8,9$. Los pares 1-2, 6-7 y 10-11 presentan similitud tanto para la L' como para su $\mathrm{F}$, por lo que no es posible individualizarlos (Ver Cuadro $\mathrm{N}^{*} 1$, Foto 1).

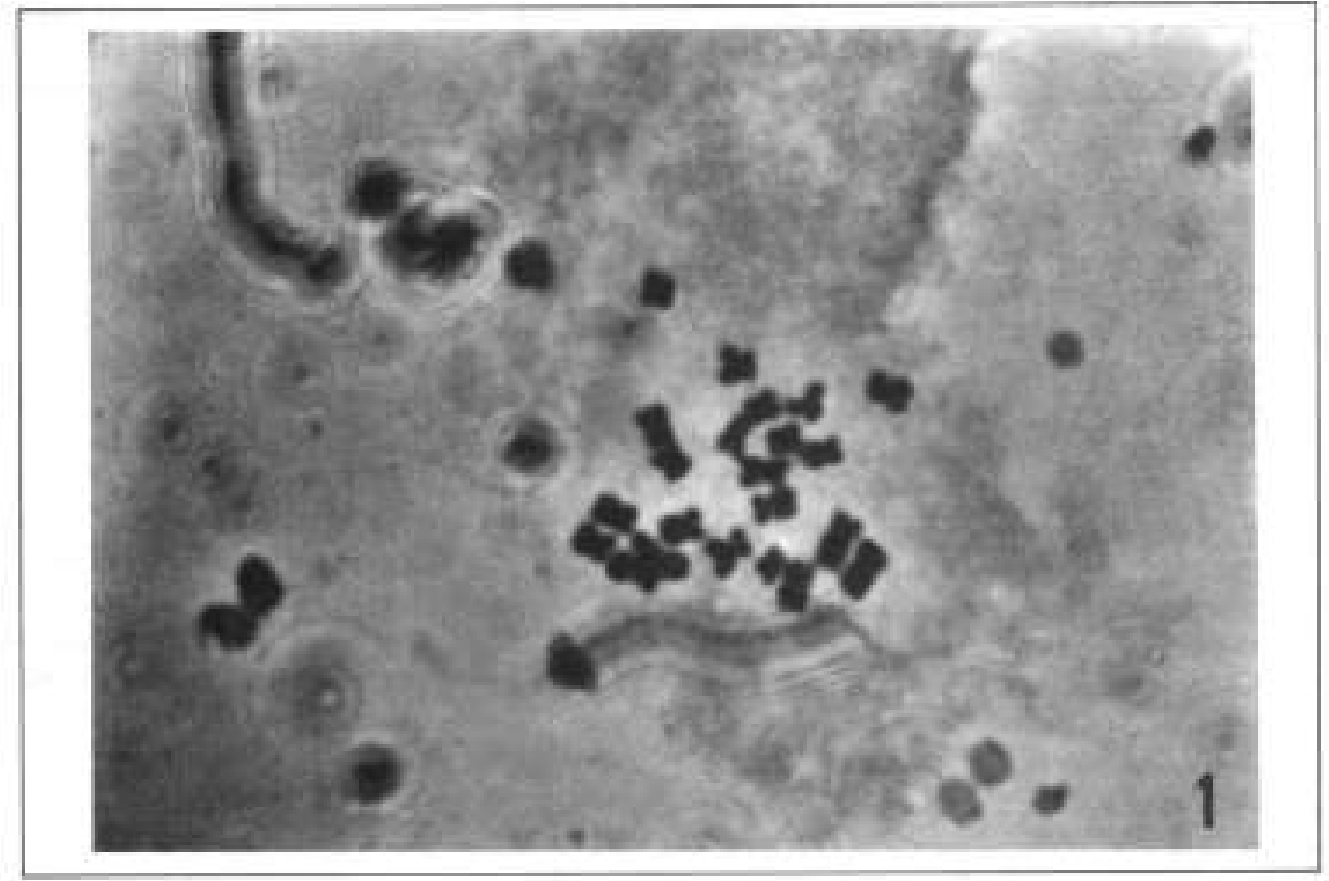

Foto 1. Metafiase de Phaseolus acutifolies Gray "Frijol tejpary" 
Cuadro $N^{\circ}$ 1. Características Cariomonfométricas de Phaseolus acutifolius.

\begin{tabular}{|c|c|c|c|c|c|}
\hline $\mathrm{N}^{\circ}$ PAR & $\mathrm{bc}(\%)$ & $\mathrm{bl}(\%)$ & $\mathrm{Lr}(\%)$ & $\mathrm{Ic}(\%)$ & TIPO \\
\hline $1^{\circ}$ & 5.02 & 6.63 & 11.65 & 43.14 & Metac. \\
\hline $2^{\circ}$ & 5.28 & 6.06 & 11.34 & 46.58 & Metac. \\
\hline $3^{\circ}$ & 4.64 & 5.29 & 9.93 & 46.77 & Metac. \\
\hline $4^{\circ}$ & 4.25 & 4.76 & 9.01 & 46.92 & Metac. \\
\hline $5^{\circ}$ & 3.63 & 4.41 & 8.04 & 45.16 & Metac. \\
\hline $6^{\circ}$ & 3.60 & 3.66 & 7.26 & 49.56 & Metac. \\
\hline $7^{\circ}$ & 3.34 & 3.92 & 7.26 & 46.26 & Metac. \\
\hline $8^{\circ}$ & 4.04 & 7.26 & 11.30 & 35.56 & Submetac. \\
\hline $9^{\circ}$ & 3.10 & 5.55 & 8.65 & 35.78 & Submetac. \\
\hline $10^{\circ}$ & 2.70 & 5.14 & 7.84 & 34.32 & Submetac. \\
\hline $11^{\circ}$ & 2.57 & 5.15 & 7.72 & 33.23 & Submetac. \\
\hline
\end{tabular}

\section{Phaseolus aureus}

Para $P h$ aureus ufrijol chinos se registró una formula cariotipica $K(n=11)=6 M+4 S M+1 A$.

Entre los cromosomas metacéntricos, los pares 1-6 presentan un indice centromérico comprendido en un rango de $43.60 \%$ para el par 4 y $47.06 \%$ para el par 3 . Respecto a las loagitudes relativas $\left(\mathrm{L}^{n}\right.$ observamos similitud para los pares 2, 3 y $4 \quad \mathrm{~L}_{2}^{\prime}=9.75 \%, \mathrm{~L}_{1}^{\prime}=9.29 \%$, $\mathrm{L}_{4}=9.02 \%$ ). Los pares 1,5 y 6 se diferencian tanto entre sí como con respecto a los demás metacéntricos $\left(\mathrm{L}_{1}=10.38 \%, \mathrm{~L}_{3}=8.24 \%, \mathrm{~L}_{\mathrm{s}}=6.92 \%\right)$.

En los 4 pares de sub-metacéntricos observados, el I* varia entre $32.45 \%$ y $33.13 \%$ para los pares 9 y 10 . respectivamente, mientras que las $L^{r}$ presentan variaciones significativas en todos los pares $\left(L^{\prime},=10.16 \%, L^{\prime},=9.43 \%\right.$, $\left.\mathrm{L}_{1}=8.36 \%, \mathrm{~L}_{10}=7.55 \%\right)$, no encontrándose ninguna similitud entre ellos.

Ell par 11 es el uninico par de cromosomas acrocéntricos registrado en todas las metafases seleccionadas en esta especie. Su caracteristica principal es su bajo indice centromérico $(I=22.68 \%)$, mientras que su longitud relativa es la más alta ( $\mathrm{L}_{\mathrm{H}}=10.92 \%$ ). Por las caracteristicas mencionadas se puede afirmar que el par 11 es un par fácilmente individualtzable en el cariotipo de $P h$ aureas.

En base a los parámetros morfonaétricos es posible identificar los pares $1,4,5,6,7,8,9,10,11$. Los pares 2 3 son los únicos que presentan similitud, tabto para su longitud relativa $\left(\mathrm{L}_{3}^{\prime}=9.75 \%, \mathrm{~L}_{1}^{\prime}=9.29 \%\right)$ como para su indice

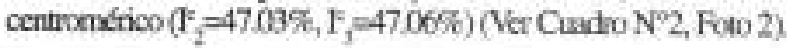

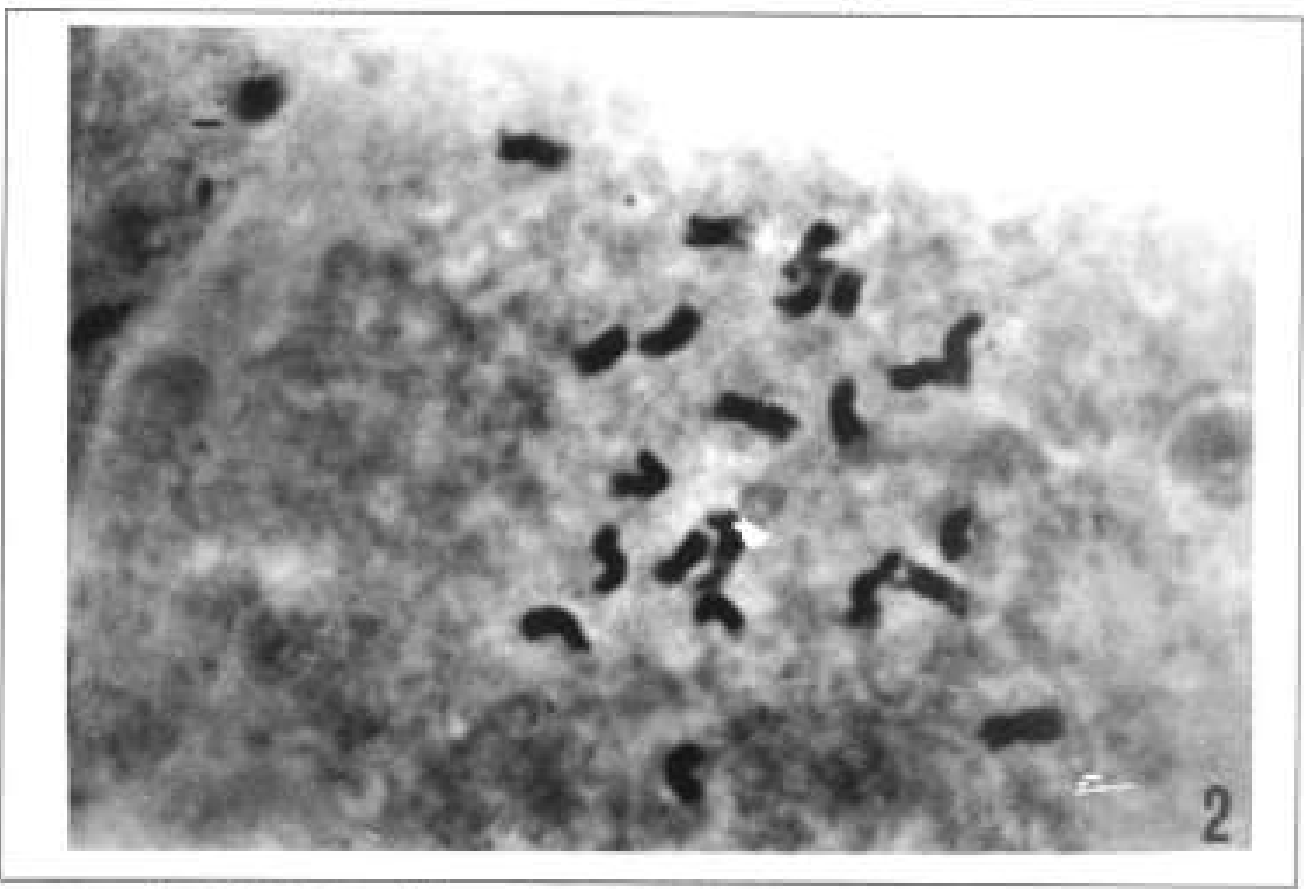

Foro 2. Meraflase de Phaseolus aureus L. (Sin.: Vigna radiata) "Frijol chino" 
Cuadro $N^{*}$ 2. Caracteristicas Cariomorfometricas de Phaseolus aurcus.

\begin{tabular}{|c|c|c|c|c|c|}
\hline $\mathrm{N}^{\circ} \mathrm{PAR}$ & $\mathrm{bc}(\%)$ & $\mathrm{bl}(\%)$ & $\mathrm{Lr}(\%)$ & $\mathrm{Ic}(\%)$ & $\mathrm{T}(\%)$ \\
\hline $1^{\circ}$ & 4.75 & 5.63 & 10.38 & 46.10 & Metac. \\
\hline $2^{\circ}$ & 4.59 & 5.16 & 9.75 & 47.03 & Metac. \\
\hline $3^{\circ}$ & 4.37 & 4.92 & 9.29 & 47.06 & Metac. \\
\hline $4^{\circ}$ & 3.93 & 5.09 & 9.02 & 43.60 & Metac. \\
\hline $5^{\circ}$ & 3.83 & 4.41 & 8.24 & 46.90 & Metac. \\
\hline $6^{\circ}$ & 3.25 & 3.67 & 6.92 & 46.94 & Metac. \\
\hline $7^{\circ}$ & 3.27 & 6.89 & 10.16 & 32.51 & Submetac. \\
\hline $8^{\circ}$ & 3.12 & 6.31 & 9.43 & 33.05 & Submetac. \\
\hline $9^{\circ}$ & 2.67 & 5.69 & 8.36 & 32.45 & Submetac. \\
\hline $10^{\circ}$ & 2.45 & 5.10 & 7.55 & 33.13 & Submetac. \\
\hline $11^{\circ}$ & 2.50 & 8.42 & 10.92 & 22.68 & Acroc. \\
\hline
\end{tabular}

Phaseolus coccineus

Se obtuvo una fómula cariotípica $K(\mathbf{n}=11)=7 \mathrm{M}+$ 4SM, que coincidió en términos generales con la registrada para $P$ h. acurifolius.

En esta especie los indices centroméricos para los pares de cromosomas metacéntricos 1-6 varían entre $43.27 \%$ y $44.70 \%$; El valor obtenido para el par 7, un cromosoma isobraquial casi perfecto $(f=48.30 \%)$, difiere significativamente de los valores de este parámetro para los demás metacéntricos. Si tomamos en cuenta las longitudes relativas observaremos similitud para los pares 1 , 2. $3\left(\mathrm{~L}_{\mathrm{t}}^{\prime}=10.99 \%, \mathrm{~L}_{2}^{\prime}=10.55 \%, \mathrm{~L}_{\mathrm{j}}^{\prime}=10.20 . \%\right)$. Los pares $4,5,6$ y 7 presentan diferencias, tanto entre si como con respecto a los demás. metacéntricos $\left(\mathrm{L}_{4}=9.33 \%\right.$, $\left.L_{s}, 8.34 \%, L_{n}^{\prime}=7.72 \% L_{+}^{\prime}=6.80 . \%\right)$.

Entre los submetacéntricos encontramos indices centroméricos que varian entre $29.38 \%$ y $32.09 \%$, para los pares 9 y 11 , respectivamente, mientras que las longitudes relativas varian significativamente para los pares 8 y $9 \mathrm{~L}_{1}=10,00.5 \mathrm{~L}_{2}=9.23$. \%) y son semejantes para los pares 10 y $11\left(\mathrm{~L}_{10}^{+}=8.60 .5 \mathrm{~L}_{11}=8.29 . \%\right)$.

Los parámetros morfométricos registrados en el Cuadro $\mathrm{N}^{\mathrm{B}} 3$ permiten identificar en términos generales los pares $1,4,5,6,7,8,9$. Mientras que los pares $2-3$ y $10-11$ presentan gran similitud, tanto para su longitud relativa como para su índice centromérico, lo que dificulta su individualización (Ver Cuadro $N^{*} 3$, Foto 3)

\section{Phaseolus lunatus}

En Ph. Iunatus "pallar se evidencio la formula cariotípica $\mathbf{K}(\mathbf{n}=11)=9 M+25 M$, obtenida del andlisis cariomorfométrico de 10 metafases.

En los 9 pares de cromosomas metacéntricos se observó un índice centromérico que varía entre $46.21 \%$ y

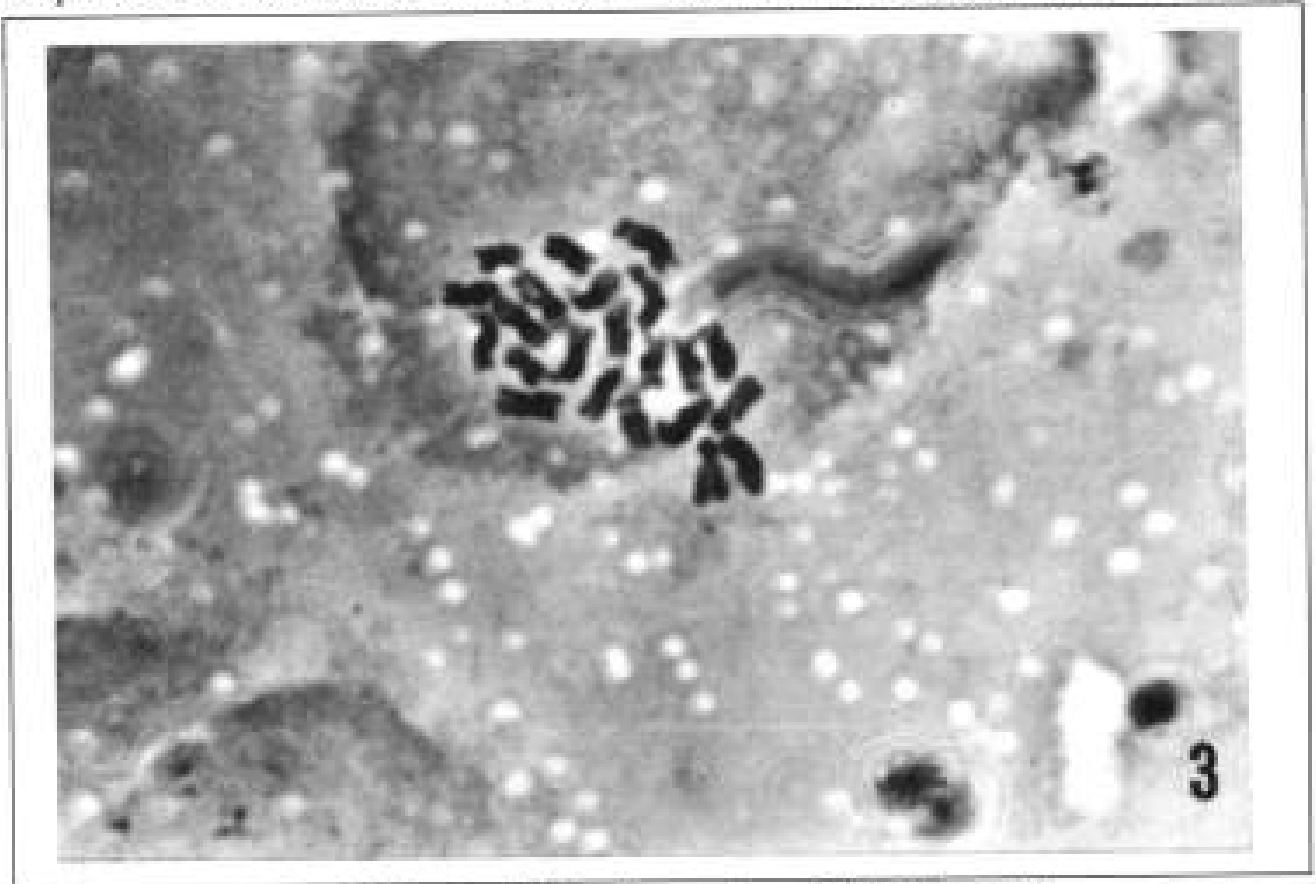

Fofo 3. Merafase de Phaseolus coccineus L "Frijol ayocote" 
Candono $N^{3}$ 3. Caracteristicas Cariomorfométricas de Phaseolus coccineus

\begin{tabular}{|c|c|c|c|c|c|}
\hline $\mathrm{N}^{\circ}$ PAR & $\mathrm{bc}(\mathcal{F})$ & $\mathrm{bl}(\mathcal{\%})$ & $\mathrm{Lr}(\mathcal{\%})$ & $\mathrm{lc}(\mathcal{F})$ & TIPO \\
\hline $\mathrm{I}^{\circ}$ & 4.89 & 6.10 & 10.99 & 44.70 & Metac. \\
\hline $2^{\circ}$ & 4.70 & 5.85 & 10.55 & 44.57 & Metac. \\
\hline $3^{\circ}$ & 4.41 & 5.79 & 10.20 & 43.27 & Metac. \\
\hline $4^{\circ}$ & 4.07 & 5.26 & 9.33 & 43.68 & Metac. \\
\hline $5^{\circ}$ & 3.68 & 4.66 & 8.34 & 43.92 & Metac. \\
\hline $6^{\circ}$ & 3.47 & 4.25 & 7.72 & 44.50 & Metac. \\
\hline $7^{\circ}$ & 3.28 & 3.52 & 6.80 & 48.30 & Metac. \\
\hline $8^{\circ}$ & 3.12 & 6.88 & 10.00 & 31.20 & Submetac. \\
\hline $9^{\circ}$ & 2.71 & 6.52 & 9.23 & 29.38 & Submetac. \\
\hline $10^{\circ}$ & 2.61 & 5.99 & 8.60 & 30.14 & Submetac. \\
\hline $11^{\circ}$ & 2.66 & 5.63 & 8.29 & 32.09 & Submetac. \\
\hline
\end{tabular}

$43.40 \%$, siendo la secuencia decreciente de variación la siguiente : $46.21 \%, 46.09 \%, 46.04 \%, 45.62 \%, 45.19 \%$, $44.34 \%, 43.76 \%, 43.66 \%$ y $43.40 \%$. para los pares 1,3 , $4,8,2,9,7,6$ y 5 respectivamente. Tomando en cuenta las longitudes relativas observaremos similitud en los pares 5.6 y $7\left(\mathrm{~L}_{3}^{\prime}=8.87 \%, \mathrm{~L}_{\mathrm{n}}=8.70 \%, \mathrm{~L}^{\prime}=8.64 . \%\right)$. Los demás pares presentan grandes diferencias, tanto entre si como con respecto a los demás metacéntricos.

En los 2 pares de cromosomas submetacéntricos, pares 10 y 11 , podemos observar variaciones significativas para ambos, tanto en su indice centromético como en su longitud absoluta $\left(\mathrm{I}_{10}{ }_{10}=32.25 \%, \mathrm{I}_{11}=35.25 \%\right.$, $L_{10}^{\prime}=10,05 \%, L_{11}^{+}=8.53 \%$ ).

Estos resultados nos permiten identificar los pares $1,2,3,4,8,9,10,11$. Los pares 5,6 y 7 presentan gran similitud en cuanto a sus parámetros morfométricos. por lo que no es posible individualizarlos en base a los mis. mos (Cuadro $\mathrm{N}^{\circ} 4$, Foto 4 ).

\section{DISCUSION}

Para iniciar el estudio cariomorfologico de $P h$. acutifolius, Ph aureas, Ph. coccineus y $P$ h. lanatus fue necesario utilizar un prefijador a fin de acumular el mayor número de metafases. Entre los inhibidores mitóticos conocidos en nuestro medio (8- hidroxiquinolina- Tjio y Levan, 1950; colchicina-O Mara, 1948 y alfa-bromonaftaleno Morrison, 1953) se escogí el alfa-bromonaftaleno por los resultados obtenidos por Turkov (1984) y Escobar (1989, 1991) en estudios cariotípicos de especies del género Phaseolus, Estos investigadores coinciden en sef̂alar la acción exitosa del alfa-beomonaftaleno a bajas iemperaturas positivas en especies cuyos cromosomas presentan longitudes menores que 4 micras.

Del análisis cariotípico en $P$ h. acutifolius, $P$ h, aureus, $P h$. cocciness y $P$ h lunaties se establece que el nùmero cromosómico de estas especies es de 22 cromosomas
$(2 \pi=22)$ en sus celulas somáticas, corrobotándose una vez más lo planteado por Kawakami (1930), Senn (1938). Berger y col. (1958), Dhaliwal y col. (1962), Thomas (1964, cit. de: Bolkhovskikh y col., 1969), Karpechenko (1925), Turkov (1984), Escobar (1989, 1991), Khairallah y col. (1991) y Bracamonte (1992). Sin embargo en el presente trabajo no se ha encontrado en ninguin caso células con $2 n=44$ como lo reportó Bolkhovskikh (1969) en $P h$. aureus, pesé a que la muestra analizada fué de 1000 células para cada especie. Por ello, nos parece posi. bie que la observación de $2 \mathrm{n}=44$ en células somáticas de esta especie se deba a errores metodológicos.

Bracamonte en 1992 propone para $P h$. Lunatur la fórmula cariotipica de $K(\mathrm{n}=11)=9 \mathrm{SM}+2 \mathrm{~A}$ en base a resultados obtenidos por técnicas de bandeamiento cromosómico y utilizando como criterios de clasificación los propuestos por Mok y Mok (1976). Sin embargo, las imágeaes y resultados presentados por Bracamonte (1992) y Mok y Mok (1976) para corroborar sus afirmaciones carecen de representatividad. Ea vista que Bracamonte no usa ninguna pre-fijación para el tratamiento de las muestras (lo que dificulta la obtención de un mayor aúmero de células metafísicas con cromosomas decondensades y distanciados) es probable que la imposibilidad de medir, identificar y realizar análisis cariotípicos exitosos y confiables. reportada en el trabajo mencionado, se deba a que simplemente no se logro estandarizat la técnica para la coloración de los ctomosomas de células somáticas de especies del géneto Phaseolus, pese a que la misma ha sido reportada detalladamente por Escobar $(1989,1991)$ y Talledo y col. (1993).

Por otro lado, las técnicas de bandeamiento cromosomico suelen ser más sofisticadas que las de coloración monocrómica. Es más, la naturaleza intrínseca de los mecanismos de coloración diferencial en cromosomas de vegetules no está clara y cada uno de los tipos de los mismos en la mayoria de los casos se denomina en base más a la técnica utilizada que a la secuencia de ubandas* obtenidas, como lo demuestra la controversia producida 


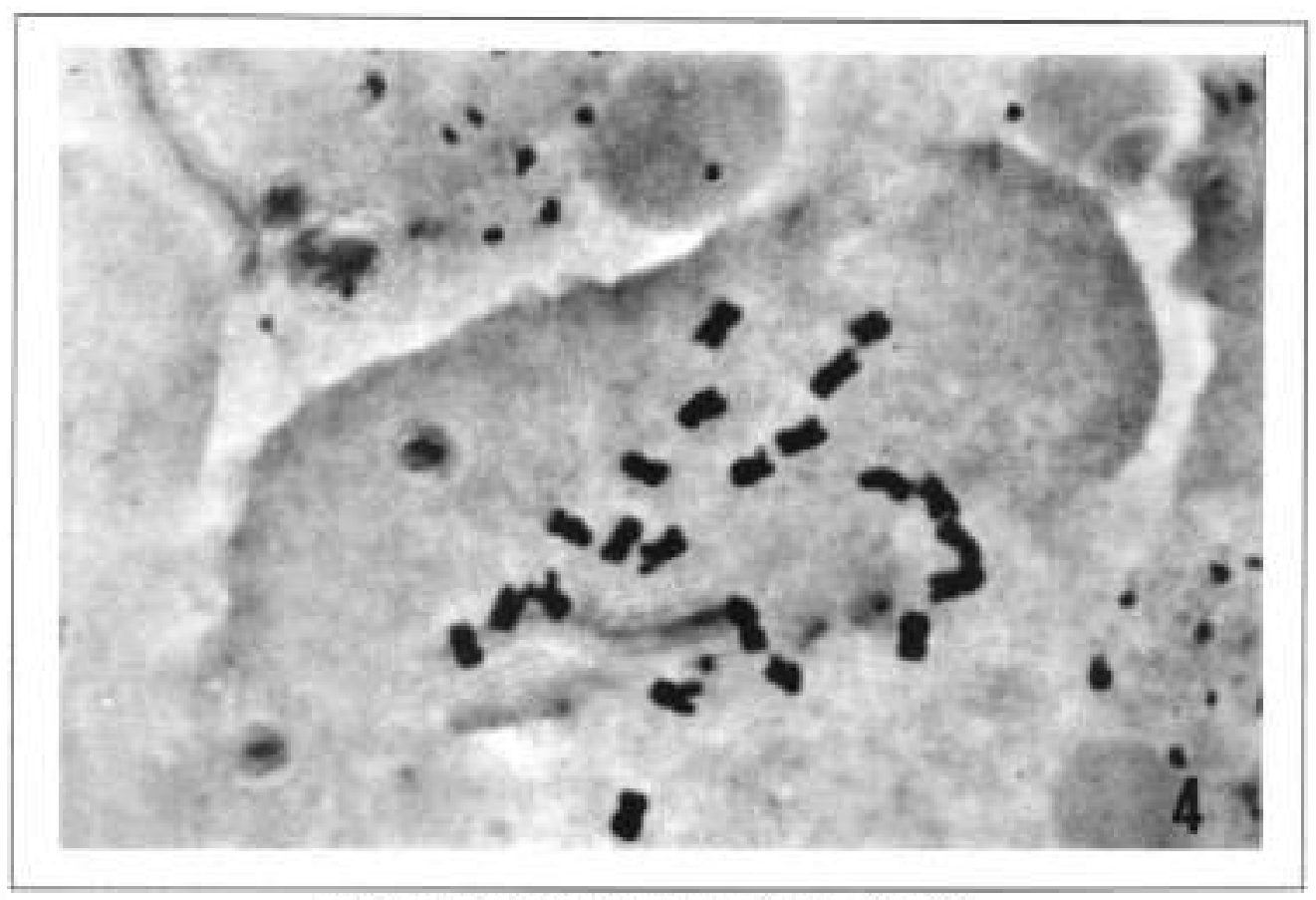

Foto 4. Metafase de Phaseolus lunatus $L$ "Pallar"

Cuadro $N^{*}$ 4. Canacteristicas Cariomorfométricus de Phaseolus lunatus.

\begin{tabular}{|c|c|c|c|c|c|}
\hline $\mathrm{N}^{\circ} \mathrm{PAR}$ & $\mathrm{bc}(\%)$ & $\mathrm{bl}(\%)$ & $\mathrm{Lr}(\%)$ & $\mathrm{Ic}(\%)$ & TIPO \\
\hline $1^{\circ}$ & 5.14 & 5.97 & 11.11 & 46.21 & Metac. \\
\hline $2^{\circ}$ & 4.58 & 5.58 & 10.16 & 45.19 & Metac. \\
\hline $3^{\circ}$ & 4.47 & 5.25 & 9.72 & 46.09 & Metac. \\
\hline $4^{\circ}$ & 4.24 & 4.97 & 9.21 & 46.04 & Metac. \\
\hline $5^{\circ}$ & 3.85 & 5.02 & 8.87 & 43.40 & Metac. \\
\hline $6^{\circ}$ & 3.79 & 4.91 & 8.70 & 43.66 & Metac. \\
\hline $7^{\circ}$ & 3.79 & 4.85 & 8.64 & 43.76 & Metac. \\
\hline $8^{\circ}$ & 3.63 & 4.35 & 7.98 & 45.62 & Metac: \\
\hline $9^{\circ}$ & 3.12 & 3.89 & 7.01 & 44.34 & Metac. \\
\hline $10^{\circ}$ & 3.24 & 6.81 & 10.05 & 32.25 & Submetac. \\
\hline $11^{\circ}$ & 3.01 & 5.52 & 8.53 & 35.25 & Submetac. \\
\hline
\end{tabular}

entre Blakey y col. (1976) y Greilbuber (1977). Por este motivo consideramos que cualquier estudio cariotipico debe ser iniciado a partir de coloraciones monocrómicas antes de intentar un abandeamientos, ya que se corre el riesgo de enfrentar problemas de resolución óptica en especies con cromosomas pequeños (2-4 u.). pretendiendo lograr una tesolución de bandas de 0.4 u, como seria el caso de las. especies del género Phaseolus.

Debido a que la nomenclatura propuesta en 1976 por Mok y Mok ha sido elaborada en forma empírica, utilizando como referencia resultados aparentemente poco representativos, en la peesente investigación hernos tomado como criterios de clasificación los propuestos por Levan y col. en 1964 y Naranjo y col. en 1983, que son los comummente aceptados.

En base a los resultados obtenidos por coloracion monocrómica y la clasificación cariotípica propuesta por Escobar (1991) se estableció por primera vez la siguiente fómula cariotipica para Ph aunetus: $K(\mathrm{~B}=11)=6 \mathrm{M}$ $+4 \mathrm{SM}+1 \mathrm{~A}$.

ParaPh buanus se propone lafómula: $K(n=11)=9 M+2 S M$.

Esta formula difiere de la propuesta por Escobar en 1989: $K(n=11)=1 M+9 S M+1 S A$, lo que se debe a que los I' obtenidos en dicho trabajo no presentan similitud con los del trabajo actual las metafases seleccionadas para este cultivo en la investigación de 1991 no presentan el mismo grado de condensación que las seleccionadas para la presente investigación, por lo que no es posible comparar los datos obtenidos.

Para $P h$, acutifolius y $P h$. coccineus se corrobora la formula cariotípica: $K(n=11)=7 M+4 S M$, planteada en 1991 por Escobar. 
Cuadro $N^{*}$ 5. Distribacioin de los Cromosomas y sus tipos por especie en ef género Phaseolus.

\begin{tabular}{|c|c|c|c|c|c|}
\hline \multirow[b]{2}{*}{ Especies } & \multicolumn{3}{|c|}{ Tipos de cromosomas } & \multirow{2}{*}{$\begin{array}{c}\text { Fórmula } \\
\text { Cariotipica }\end{array}$} & \multirow[t]{2}{*}{ Simetría } \\
\hline & $\mathrm{M}(\%)$ & $\mathrm{SM}(\%)$ & $A(\%)$ & & \\
\hline Ph. acutifolius & 63.64 & 36.36 & 00.00 & $\mathrm{~K}=7 \mathrm{M}+4 \mathrm{SM}$ & ++ \\
\hline Ph. aureas & 54.55 & 36.36 & 9.09 & $\mathrm{~K}=6 \mathrm{M}+4 \mathrm{SM}+1 \mathrm{~A}$ & $+\cdot$ \\
\hline Ph. coccineus. & 63.64 & 36.36 & 00.00 & $\mathrm{~K}=7 \mathrm{M}+4 \mathrm{SM}$ & ++ \\
\hline Ph, lenatus & 81.82 & 18.18 & 00.00 & $\mathrm{~K}=9 \mathrm{M}+2 \mathrm{SM}$ & +++ \\
\hline
\end{tabular}

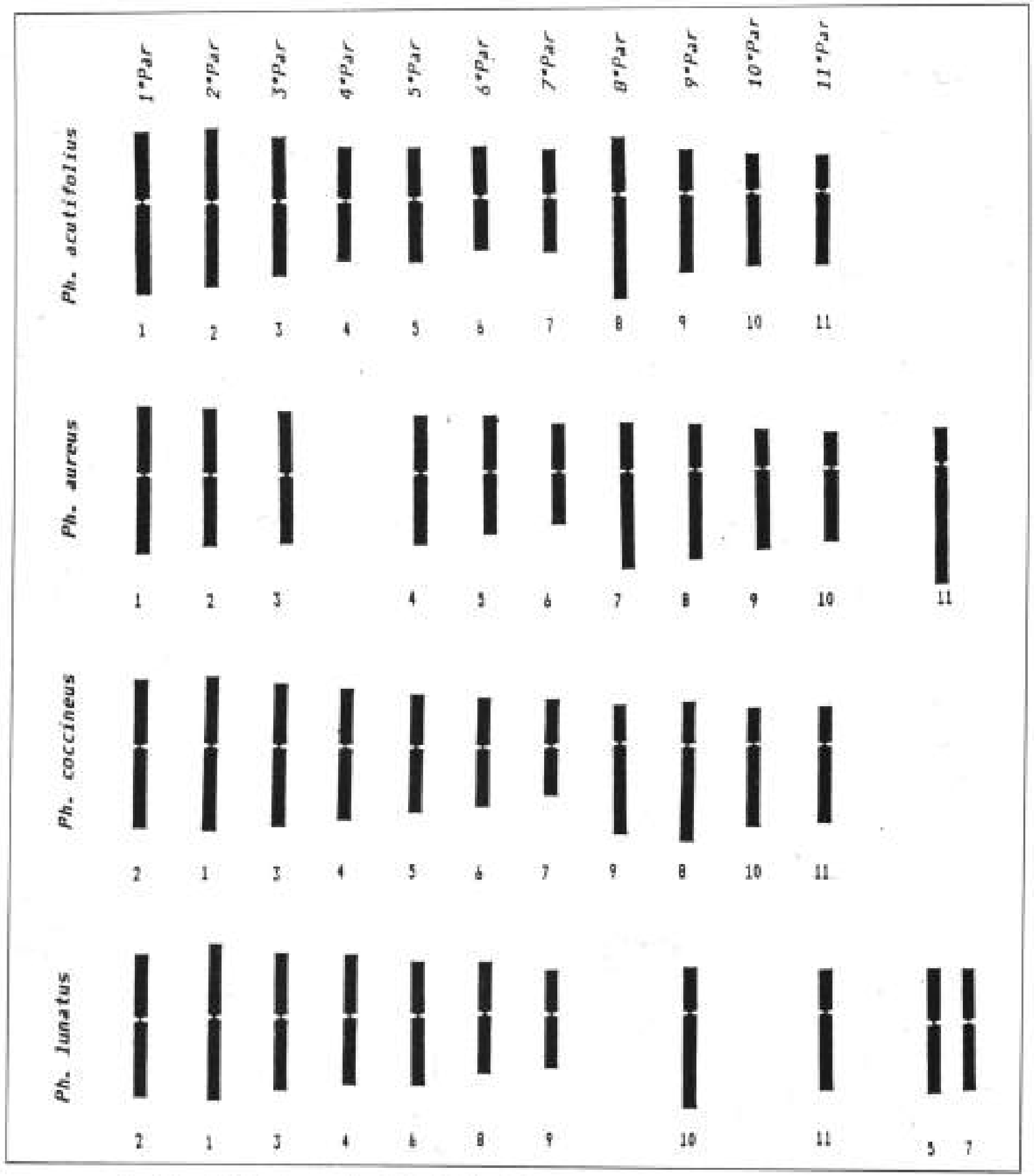

Fig. $N^{\circ}$ L. And́lisis Companativo de la Morfologia Cramosómica en Especies del Género Phaseolus. Se represention los idlogramas de las 4 especies, tomaedo camo referencia el de Ph. acutiofolius. Nobere la "homealogia' entre algunos cromonomas y las diferencias entre otros. 
La interpretación de las fónmulas cariotípicas y los resultados obtenidos del análisis cariotipico comparativo en $P$ h, acutifolius y Ph, coccineus, $K(n=11)=7 \mathrm{M}+4 \mathrm{SM}$. con cromosomas metacéntricos en un 63.64 \% y sub. metacéntricos en un $36,36 \%$; Ph. aureus, $\mathrm{K}(\mathrm{n}=11)=6 \mathrm{M}$ $+4 \mathrm{SM}+\mathrm{IA}$, con cromosomas metacéntricos en un $54,54 \%$, resolución de bandas de 0.4 u., como sería el caso de las especies del género Phaseolas:

Debide a que la nomenclatura propuesta en 1976 por Mok y Mok ha sido claboradaen forma empitica, utibzando como referencia resultados aparenteneste poco represen. tativos, en la presene investigación hemos tomado como criterios de clasificación los ptopuestos por levan y col. en 1964 y Naranjo y col en 1983, que son los comunnente aceptados.

En base a los resultados obtenidos por coloración monocrómica y la clasificación cariotípica propuesta por Escobar (1991) se estahleció por primera vez la siguiente fórmula cariotipica para Ph.carous: $K(n=11)=6 \mathrm{M}$ $+4 \mathrm{SM}+1 \mathrm{~A}$

Para $P h$. Lunahes se propone la fórmula: $K(n=11)$ $=9 \mathrm{M}+2 \mathrm{SM}$

Esta fómula difiere de la propuesta por Escohar en 1989: $\mathrm{K}(\mathrm{a}=11)=1 \mathrm{M}+9 \mathrm{SM}+1 \mathrm{SA}$, lo que se debe a que los $\mathrm{F}$ obtenidos en dicho trabajo no presentan similitud con los del trabajo actual: las metafases seleccionadas para este cultivo en la investigación de 1991 no presentas el mismo grado de condensación que las seleccionadas para la presente investigación, por lo que no es posible comparar los datos obtenidos:

Para Ph. arutifolias y Ph. cecciness se corrobora lit fórmula cariotipica: $K(n=! 1)=7 \mathrm{M}+4 \mathrm{SM}$, planteadá en 1991 por Escobar.

La interprefación de las fórmulas cariotipicas y los resultaklos obtenides del andlisis canotipico comparativo en Ph. acutifolius y Ph. coccineus, $\mathrm{K}(\mathrm{n}=11)=7 \mathrm{M}+4 \mathrm{SM}$. cont cromosomas metacéntricos en un $63.64 \%$ y submetacentricos en un $36.36 \% ; P h$. aicreis, $K(n=11)=6 \mathrm{M}$ $+4 S M+1 A$, con cromosomas metacéntricos en un $54,54 \%$, sub-metacéntricos en un $36,36 \%$ y acrocétricos en un $9.09 \%$ y $P$ h. funatar, $K(n=11)=9 \mathrm{M}+2 \mathrm{SM}$. con $81.82 \%$ de cromosomas metacéntricas y un $18.18 \%$ de sab-metacéntricos; nos permiten establecer usa alta simetría de los cariotipos de las especies estudiadas, coincidiendo con lo propuesto en 1991 por Escobar para la

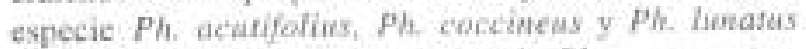
«pallaro, Lat simetria del carionipo de Ph. aureas se reporta por primera wez. (Cuadro $\mathrm{N}^{3}$ 5, Fig. $\mathrm{N}^{2}$ ) ).

\section{CONCLUSIONES}

1. El estudio morfológioo y comparatio nos ha permitido establecer diferencias de tamaño a nivel citológico, ob- servándose que las cellulis de las diferentes especies presentan variaciones significativas de tamaño.

2.- La investigación caniotipica corrobora unà vez más en las especies estudiadas la existencia de 22 cromosomas $(2 \mathrm{n}=22)$ en sus células somáticas.

3.- Se han determinado las siguientes fórmulas cariotipicas:

$$
\begin{array}{ll}
\text { Ph. acutifolias } & K(n=11)=7 M+4 S M \\
\text { Ph. aureis } & K(n=11)=6 M+4 S M+1 A . \\
\text { Ph. coccineus } & K(n=11)=7 M+4 S M \\
\text { Ph, ianatus } & K(n=11)=9 M+25 M .
\end{array}
$$

4. El análisis cariomocfológico establece cariotipos altamente simétricos para lodas las especies, siendo esta simetria mayor en Ph, acurifoliss, Ph. coccineus (cromosamas metacéntricos 63.64\% y sub-meracéntricos $36.36 \%$ ) y $P h$. linatus (ctomosomas metacéntricos $81.82 \%$ y cromosomas sub-metacéntrices $18.18 \%$ ) con un porcestaje de simetria jgual a $100 \%$ en sus células somäticas, mientras que en $P h$ aurras este valot es del $91 \%$ (cromosonas metacéntricos $54,54 \%$ y cromcsomas sub-metacéntricos 36,365 ).

\section{AGRADECIMIENTOS}

Los autores expresamos nuestro reconocimiento al CONCYTEC (Contrato Ne 881-12-93), at Programa Colaborativo COTFSU - CIP, al INIA-Ica y a la Universidad Ricardo Palma, Vice-Rectorado Académico. Facultad de Cuencias Biológicas y Departamento Académico de Ciencias, sin cuyo apoyo no hubiera sido posible desarrollar el presente trabajo.

\section{REFERENCIAS BIBLIOGRAFICAS}

BLAKEY, D, ; Fll.ION, G. 1976. "Differential Giemsa staining in plantso. Chromosorna (Berl) 56:191-197.

BOLKHOVSKIKH, Z; GRIF, V; MATVIEYEVA,T ; ZAKHARYEVA, O. 1969. "Chromosome numbers of flowering plantss I In Ed. Editorial Nauika. Leningrado. Moscú 520 pp.

BRACAMONTE, 0.0.1992, aCitogenetica de Phaseolus vwlgarisn. Tesis para. Magister Scientiae en la especia. lidad de Mejoramiento Genétieo de Plantas, 76 pp. UNALM. Lima-Peru.

ESCOBAR G, C, 1989, westudio comparativo de tres lineas de cultivo peruanas de Phaseolus lanafies L. upallarow. Tesis para... Bxchiller en Biologia, 66 pp. Univ. Ricardo Palma, Lima. 
ESCOBAR G.,C. 1991, "Estudio cariotípico comparativo en cuatro especies del género Phaseolus: Ph. acutifolius Gray, efrijol tepary», $P$ h, coccineus $\mathrm{L}$, efrijol ayocotes, Ph. lunatus $\mathrm{L}$, spallars y $P$ h. valgaris $\mathrm{L}$. efrijolcomún:s. Tesis para optar el titulo de Licenciado en Biologia, 86 pp. Univ. Ricardo Palma. Lima

ESCOBAR G.,C ; TALLEDO, D. Y VILLACORTA, J. 1992. aAnalisis cariotípico en metafase mitctica en tres líneas de cultivo peruanas de Phascolus lunatus $\mathrm{L}$. "Pallars. Boletín de Lima N* 83:19-22.

GREILBUBER, J. 1977. *Why plant chromosomes do not show G- bands\%. Theor.Appl.Genet. 50:121-124.

HOLLE, M. 1984, Recursos Genéticos Vegetales de América del Sur: Pasado, Presente y Futuro. PP. 7-19. En: Anales del Simposio de Recursos Fitogenéticos. Consejo Internacional de Recursos Fitogenéticos, Valdivia. Chile. $151 \mathrm{pp}$.

KARPECHENKO, G.D. 1925, eTrudy po prikls. Bot:; Genetike y Sel. T.14. Byp 2:143-148.

KHAIRALLAH, M.M.; ADAMS, M W; SEARS, B.B. 1991. Mitochondrial genome size variation and restriction fragment length polymorphisms in three Phaseolus species. Theor. Appl. Genet. 82:321-328.

LEVAN, A.; FRIEDGA,K.; SADBERG, K. 1964. Nomenclature for centromeric position on chromosomesw, Hereditas 52:201-220.

MOK, D.W.S. \& MOK, M.C. 1976. «A modified Giemsa. technique for identifying Bean chromosomess. J. Hered. $67: 187-188$
MORRISON, J.W, 1953, «Chromosome behaviour in wheat monosomicsw, Heredity 7:203-217.

NARANJO, C.; POGGIO, L \& BRANDHAM, P. 1983. *A practical method of chromosome classification on the basis of centromere positions, Genet, 62:51-53,

OMARA, J.G. 1948. «Acetic acid methods for chromosome studies at prophase and metaphase in meristems», Stain Technol. 23:201-204.

SASAKI, M. 1961. *Observations on the modification in size an shape of chromosome due to technical procedure\%, Chromosoma 11:514-522.

TALLEDO, D.; ESCOBAR,C; ALLEMAN, V, 1993، \&Introducción al analisis cromosómico en vegetalesn. Univ, Ricardo Palma, Lima-Perú, 14 Ipp.

TJO, J.H.: LEVAN, A. 1950, $\propto$ The use of oxyquinoline in chromosome analyses», An. Estac, Exp. Aula Dei, 2, 21.

TJIO, J.H.: LEVAN, A. 1954, «Some experiences with acetic-orceina. An. Estac. Exp. Aula Dei, 3:225-228.

TURKOV, V.; GUZHOV, YU. L.; SHELEPINA, G.; YLIYANARASHI, S. 1984, CCromosomas de las plantas cultivadas y de sus predecesores silvestres $\propto$. Univ. de fa Amistad de los Pueblos Patricio Lumumba Edit. UDN. Moscú 70 pp.

TURKOV, V; GUZHOV, YU, L.; SHELEPINA, G.; YLIYANARASHI, S. 1988. "Las investigaciones cromosómicas en vegetales en los problemas del fitomejoramiento, Ingeniería Celular y Monitoreo Genéticos. Univ, de la Amistad de los Pueblos Patricio Lumumba. Edit. UDN. Moscú 64 pp. 